\title{
Anticoagulation in Pregnant Women with Mechanical Heart Valve Prostheses: 25-Year Experience at a Tertiary Care Hospital in a Developing Country
}

\author{
Ahmed Tounsi1 ${ }^{*}$, Dorra Abid'1, Doulira Louati2 ${ }^{2}$, Souad Mallek¹, Malek Akrout1, Leila Abid1, \\ Mohamed Abdennadher ${ }^{3}$, Imed Frikha ${ }^{3}$, Kais Chaabene ${ }^{2}$, Mourad Hentati ${ }^{1}$, \\ Samir Kammoun 1 \\ ${ }^{1}$ Cardiology Department, Hedi Chaker Hospital, Sfax, Tunisia \\ ${ }^{2}$ Obstetrics and Gynecology Department, Hedi Chaker Hospital, Sfax, Tunisia \\ ${ }^{3}$ Department of Cardiovascular Surgery, Habib Bourguiba Hospital, Sfax, Tunisia \\ Email: ${ }^{*}$ ahmed.tounsi2@gmail.com
}

Received 31 March 2014; revised 9 May 2014; accepted 16 May 2014

Copyright (C) 2014 by authors and Scientific Research Publishing Inc.

This work is licensed under the Creative Commons Attribution International License (CC BY).

http://creativecommons.org/licenses/by/4.0/

(c) (i) Open Access

\begin{abstract}
The aim of this retrospective study was to compare the different anticoagulation regimens used in pregnant women with prosthetic heart valves. We reviewed 86 pregnancies in 57 women from 1987 to 2011. The patients were divided into two groups: group A (39 pregnancies) had oral acenocoumarol throughout pregnancy; and in group B (47 pregnancies), acenocoumarol was replaced by subcutaneous heparin during the first trimester. Both groups received heparin at the time of delivery. The valves replaced were mitral (59.65\%), aortic $(12.28 \%)$, or both $(28.07 \%)$. 74 pregnancies $(86.04 \%)$ resulted in live births, $9(10.46 \%)$ had stillbirths, 1 (1.16\%) had spontaneous abortion and $2(2.32 \%)$ underwent therapeutic abortions. The live birth rate was higher in women on heparin $(87.23 \%)$ compared with those on acenocoumarol $(84.61 \%)$. No malformations appeared in the 74 newborns, except for one case of hydrocephalus. There was one maternal death due to acute mitral valvular thrombosis while on heparin in the first trimester. Hemorrhagic complications occurred in 13 patients in the postpartum period, 4 of whom required transfusion. No anticoagulant regimen can be said to be entirely safe for use during pregnancy as there is a degree of risk with each regimen. Heparin does not offer a clear advantage over oral anticoagulation in the pregnancy outcome.
\end{abstract}

\section{Keywords}

Anticoagulation, Pregnancy, Mechanical Heart Valve Prostheses, Maternal Complications, Heparin,

"Corresponding author.

How to cite this paper: Tounsi, A., et al. (2014) Anticoagulation in Pregnant Women with Mechanical Heart Valve Prostheses: 25-Year Experience at a Tertiary Care Hospital in a Developing Country. World Journal of Cardiovascular Diseases, 4, 287-293. http://dx.doi.org/10.4236/wjcd.2014.46037 


\section{Acenocoumarol}

\section{Introduction}

The prevalence of women with mechanical valves becoming pregnant has been on the rise, especially in developing countries where the incidence of valvular diseases in children is still high.

Many of them undergo valve replacement surgeries at a young age; subsequently they get pregnant. Patients with mechanical prosthetic valves require lifelong anticoagulation to minimize the high risk of associated thrombo-embolic complications. Being a physiologic hypercoagulable state, pregnancy further increases the risk of such events [1]. Even though there is an agreement regarding the use of mandatory therapeutic anticoagulation in pregnancy for these women, there is no agreement on the safest and the most effective method of anticoagulation.

Treatment options include oral anticoagulants (coumarin derivatives), unfractionated heparin (UFH) and low molecularweight heparin (LMWH). The choice of anticoagulation strategy needs to be taken after thoroughly balancing the pros and cons of the various strategies individually in each patient. The risk of thromboembolic complications in women on coumarin derivatives is reduced to $3.7 \%$, if they are maintained on it throughout pregnancy, with a $0.5 \%-1.8 \%$ risk of maternal death [2]-[4]. However, its use must be tempered by its probable adverse effects on the fetus with a reported $12 \%$ rate of late fetal loss and $6.4 \%$ rate of congenital malformations [3] [5]. This has led many authorities to advocate the use of heparin as a replacement for coumarin derivatives, at least during the first trimester. One drawback of UFH is the high rate of treatment failure with reports of maternal mortality from thrombosed valves [3] [6].

The aim of our study is to analyze the maternal and perinatal outcome in these high risk pregnancies and also, to evaluate different anticoagulation regimes used in pregnant women with prosthetic heart valves.

\section{Materials and Methods}

All pregnant women with prosthetic heart valves registered at our hospital during the time period from January 1987 to December 2011 are included in the study. This study received ethical approval from the Ethics Committee our university hospital and informed consent was obtained.

Information on maternal age, parity, valve replacement procedure, anticoagulation regime, gestational age at delivery, mode of delivery, thrombo-embolic and obstetric complications (abortions, still birth, preterm delivery, intrauterine growth restriction (IUGR) or hemorrhagic complications) and the neonatal data (congenital malformations, birth weight and neonatal morbidity ) were reviewed. Thrombo-embolic complications were defined as peripheral arterial embolism or valve thrombosis or embolism manifested by a transient or permanent neurologic deficit. Hemorrhagic complications were defined as the occurrence of postpartum hemorrhage, hematuria and vulvovaginal hematoma. Spontaneous abortion was defined as any spontaneous fetal loss before 20 weeks of gestation. Therapeutic abortions included all medically indicated terminations before 20 weeks of gestation. Still birth referred to fetal loss at gestation more than 20 weeks. Preterm delivery was defined as birth before 37 weeks of gestation. All babies born with a birth weight less than 2 standard deviation ( $<10$ th percentile) as per the institutional birth weight curve reference were classified as IUGR.

The two regimes followed in our hospital were offered: 1) Continuation of oral anticoagulants (acenocoumarol) till 36 weeks gestation because the pregnancy was confirmed too late; Or 2) Change over to unfractionated heparin in the first trimester when the pregnancy was confirmed (till 12 weeks), switching back to oral anticoagulants in second trimester which is continued till 36 weeks of gestation. After 36 weeks, they were switched over to unfractionated heparin again till delivery. This was done earlier in the presence of obstetric complications which necessitated delivery at an earlier gestation in both the regimes. Unfractionated heparin was started at a dose of 20,000 to 24,000 units/day (according to the body weight) subcutaneously with twice/thrice a day dosing and was titrated to attain a targeted Activated Partial Thromboplastin Time (APTT) 2 - 3 times the control. Acenocoumarol was started at a dose of $2 \mathrm{mg} /$ day and later titrated to attain a targeted International Normalized Ratio (INR) of 2.5 - 3.5. Women who opted for the second regime were hospitalized till the targeted APTT levels were achieved and then oral therapy was restarted on outpatient basis in the second trimester. 
In both groups, oral acenocoumarol was stopped 2 weeks before the expected date of delivery, switching to subcutaneous heparin with a target APTT of twice the normal level.

Induction of labor was done for obstetric indications or at 38 weeks for planned delivery. Heparin was discontinued at the onset of labor or prior to induction of labor and was restarted $6 \mathrm{~h}$ after delivery. Heparin was restarted at the same dose before discontinuation and titrated according to the APTT value.

Within the first 2 days after delivery acenocoumarol was added and the heparin was discontinued when an INR of 2 or greater was achieved.

Women were grouped into 2 groups according to the anticoagulation regimes they received in the first trimester: 1) Group A received acenocoumarol, 2) Group B received heparin. Data was analyzed using SPSS software 18.0. Outcome, variables were compared by using Student t-test and Chi-square test or Fisher exact test. A p-value less than 0.05 was considered significant.

\section{Results}

Over a 25-year period, we identified 86 pregnancies in 57 women with prosthetic heart valves. Maternal and disease characteristics are summarized in Table 1. Twenty three women had more than one pregnancy (20 women had two pregnancies, one had three pregnancies, one had four pregnancies and one had five pregnancies). There was a mean (SD) interval between surgery and pregnancy of 5.48 (4.9) years (range 0.5 to 19). Most of the women underwent replacement of the mitral valve (59.65\%). Most of the valves were of bileaflet type (43/73; 58.9\%), 10 (13.7\%) were tilting disc valves, and 20 (27.4\%) were ball cage valves.

The choice of prosthesis was a matter of the surgeon's preference (Patients with bioprosthetic valves were excluded from the study). None of the patients refused anticoagulant therapy.

Acenocoumarol was given in 39 (45.35\%) pregnancies (group A) and heparin in 47 (54.65\%) pregnancies (group B) in the first trimester. We compared the obstetric outcome and complications in the two groups. Table 2 and Table 3 depict the comparison of the perinatal outcome in these women. Overall, 74 (86.04\%) pregnancies resulted in live newborn infants, 9 (10.46\%) had still births, 1 (1.16\%) had spontaneous abortion while 2 (2.32\%) had a therapeutic abortions.

Table 1. Maternal characteristics of 57 women with mechanical prosthetic valves.

\begin{tabular}{cc}
\hline Variables & $\mathbf{N}=\mathbf{5 7}$ \\
\hline Age (years) & $31.3+/-5.11$ \\
Age at surgery & $24.67+/-6.04$ \\
Gravidity & $2.3[1-8]$ \\
Parity & \\
Nulliparous & $38(66.67 \%)$ \\
Multiparous & $19(33.33 \%)$ \\
Valve site & \\
Mitral & Aortic \\
Mitral and aortic & $34(59.65 \%)$ \\
Valve type & $7(12.28 \%)$ \\
Ball caged valves & $16(28.07 \%)$ \\
Tilting disc & \\
Bileaflet valves & $20(27.4 \%)$ \\
\hline
\end{tabular}

Table 2. Maternal complications.

\begin{tabular}{cccc}
\hline variables & Group A $(\mathbf{n}=\mathbf{3 9})$ & Group B $(\mathbf{n}=\mathbf{4 7})$ & P value \\
\hline Valve thrombosis & 1 & 6 & 0.08 \\
Thromboembolic episode & 4 & 2 & 0.27 \\
Hemorrahagic complications & 8 & 5 & 0.2 \\
Infective endocarditis & 2 & 5 & 0.35 \\
mortality & 0 & 1 & 0.25 \\
\hline
\end{tabular}


Table 3. Comparison of perinatal outcome of different regimes of anticoagulation.

\begin{tabular}{cccc}
\hline variales & Group A (n= 39) & Group B (n= 47) & P value \\
\hline Outcome & 1 & & 0.67 \\
Abortion & 33 & 2 & 0.72 \\
Live birth & 5 & 41 & 0.51 \\
Still birth & 8 & 5 & 0.2 \\
Preterm delivery & $2612+/-489$ & $2681+/-676$ & 0.59 \\
Birth weight (grams) & 11 & 14 & 0.83 \\
IUGR & $36.23+/-3.6$ & $36.43+/-5.14$ & 0.83 \\
Gestation at delivery (weeks) & 1 & 0 & 0.18 \\
\hline
\end{tabular}

Therapeutic abortions were done in the case of 2 women with advanced NYHA cardiac class III or IV. Of the nine still births, 5 occurred in group A (unexplained still birth) and 4 in group B.

After excluding the abortions, deliveries were by cesarean section in $39.76 \%(\mathrm{n}=33)$ and vaginal in $60.24 \%$ $(n=50)$ of cases, $16 \%(n=8)$ of which were operative vaginal deliveries.

Indications for cesarean sections were breech presentation not willing for vaginal birth $(\mathrm{n}=12)$, failure to progress $(n=6)$, fetal distress $(n=8)$ and others including bad obstetrics history and unstable lie $(n=7)$. The mean gestational age at delivery was $36.35+/-4.5$ weeks with a mean birth weight of $2656+/-593$ g. The incidence of IUGR and preterm delivery is not found to be different among the groups ( $>>0.05$ ). One baby developed a hydrocephalus.

The mother of that baby received oral anticoagulants during the first trimester. We are not certain about the origin of the hydrocephalus but we cannot rule out intracranial bleeding as a cause. There was one neonatal death which happened in patient who had received acenocoumarol till 36 weeks. She delivered at 37 weeks, a baby boy weighing $3.1 \mathrm{~kg}$ who expired in the second hour of life.

No malformations were diagnosed in the remaining 32 newborn infants who were exposed to oral anticoagulants during the first trimester (Table 3). The mean (SD) daily dose of acenocoumarol at the beginning of pregnancy was $3.58(1.27) \mathrm{mg}$, range 0.66 to 6.5 . In 6 cases the daily dose was $5 \mathrm{mg}$ or more.

The mean gestation at which the women were switched over to heparin was $5.86+/-1.68$ weeks in the first trimester (in group B) and $35.32+/-2.22$ weeks in the third trimester.

Comparison of the occurrence of maternal complications during the antenatal and the peripartum period between the two groups is shown in Table 2.

Hemorrhagic complications in the form of hematuria during the antenatal period, vaginal hematoma which required evacuation and postpartum hemorrhage (managed conservatively) occurred in 5 women in group B while 8 women in group A had secondary post-partum hemorrhage. The incidence of hemorrhagic complications was found to be higher when the patients were on acenocoumarol in the peripartum period; 2 of them required blood transfusions. Women who developed postpartum hemorrhage (primary and secondary) had INR values. There was one maternal death in group B (presented with acute heart failure in first trimester while on heparin and was found to have acute mitral valvular thrombosis).

\section{Discussion}

The management of anticoagulation during pregnancy is controversial and there is no ideal treatment. Patients with prosthetic heart valves have the highest thromboembolic risk, and pregnancy itself is a thrombogenic situation owing to physiological hypercoagulation. Modern valves have fewer thromboembolic complications because of better design and materials, but there are many women with earlier models of prosthetic valve attending our anticoagulation clinic who become pregnant.

The present study presents the experience with different anticoagulant regimes used in a tertiary centre from a developing country. Overall, 86.04\% resulted in live births and 60.24\% had vaginal delivery. There was one maternal death due to acute valvular thrombosis.

Pregnancy induces derangements in the hemostatic mechanism predisposing these women to thrombo-embolic complications [1]. 
Increased concentration of circulating clotting factors, faster platelet turnover and reduced fibrinolytic activity contribute to a hypercoagulable state. These changes along with the presence of mechanical valves are associated with increased risks of thromboembolic risks.

Pregnancy in women with mechanical heart valves remains a challenge to the obstetrician and the cardiologist. The major concern regarding the treatment during pregnancy is the teratogenic effect of the different anticoagulants on the fetus and the risk of thrombo-embolic complications for the mother.

The risk of valve thrombosis relies on many factors, including type, number, and position of the valve with higher risk in mitral valves, arrhythmias, previous thrombosis, and adequacy of anticoagulation [7]. Newer valve types like bileaflet St. Jude valves are reported to have a lower thrombogenic potential [3]. Despite that, 4 of our valve thromboses occurred in women with such valves, all of which were mitral valve prostheses.

According to the American Heart Association and the American College of Cardiology, if UFH or LMWH is to be used in pregnant women with heart valve prostheses, they should be administered in adequate dosages and monitored at least twice weekly [8]. Though the better bio-availability, non-teratogenicity (as it does not cross the placenta) and less hemorrhagic complications with LMWH make it a potential alternative, American College of Obstetricians and Gynecologists (ACOG) continues to warn against the use of LMWHs in these patients because of reports of associated valve thrombosis and it also requires anti-Xa monitoring in pregnancy [6] [9]. Reports regarding the optimal therapy of these women during pregnancy vary in different parts of the world with respect to the regimes used, involving oral anticoagulants and the UFH. In Tunisia and other countries with poor socioeconomic conditions, compliance is hampered by the fact that heparin requires 2 to 3 injections every day. It is difficult, if not impossible, for a villager to travel a long distance to receive the injection. It would be more practical for her to use tablets.

Our 3.7\% risk of thromboembolism is comparable with that cited in a large series and a systematic review in patients maintained on warfarin throughout pregnancy [2]-[4]. This might be due to the high percentage of patients who continued to receive warfarin beyond the first trimester (85.7\%). Despite following a standard protocol of shifting patients from warfarin to heparin at term, hemorrhage requiring transfusion complicated 4 cases before or shortly after the shift was started.

Contrary to the report in our series, the incidence of hemorrhagic complications was higher in the patients on heparin and was found to be statistically different between the groups. In our study, hemorrhagic complications occurred more frequently when patients were on acenocoumarol.

However, we should consider the fact that all the patients on coumarin had been switched to heparin at about 36 weeks of gestation, and discrimination would be difficult. On the other hand, in concordance with our data, Sbarouni's collaborative study reported more thromboembolic and bleeding complications in women treated with heparin than those who were maintained on oral anticoagulants [10].

Heparin, a macromolecular agent, does not cross the placental barrier and offers theoretical advantages over Coumadin. Heparin avoids fetal risk but is somewhat difficult to manage. On the other hand, oral anticoagulants are easy to administer but it may cause embryopathy, and central nervous system abnormalities. In order to prevent possible teratogenic effects, heparin is suggested to be substituted for Coumadin before the most vulnerable period, embryogenesis. Coumarin derivatives, when given between the 6th and the 12th week of pregnancy carry the risk of embryopathy which is found to be associated with abortions and late fetal loss [2] [11] [12].

Both oral anticoagulants and heparin carry the risk of fetal loss, probably from different mechanisms. Heparin can induce retroplacental hemorrhage; although its administration during the first trimester can prevent the occurrence of malformations. In the present series, the spontaneous abortion occurred in woman receiving UFH in the first trimester. The value of replacing oral anticoagulants with heparin in the first trimester to decrease the spontaneous abortion rate is not substantiated in newer studies [3] [13].

The association between the dose of oral anticoagulant and embryopathy has been proposed; a dose more than $5 \mathrm{mg}$ is associated with adverse effects [14]. In a series by Vitale et al. [2], about $50 \%$ of the patients received higher dose of warfarin, with an associated fetal loss of $76 \%$. In another study, it was $22.2 \%$ with a $9.7 \%$ associated adverse outcome [12]. The equivalent dose of acenocoumarol to $5 \mathrm{mg}$ warfarin is approximately $2.5 \mathrm{mg}$ as shown in a study by van Leeuwen et al. [15]. Although the in present study the mean dose was $3.56+/-1.16$ $\mathrm{mg} / \mathrm{day}$, no congenital malformations have been reported. We had one case of hydrocephalus in a baby born to a woman exposed to oral anticoagulants during her entire pregnancy; her baby was delivered while she was still on coumarins. We cannot be certain about the cause of the hydrocephalus but intrauterine or perinatal intracranial haemorrhage cannot be ruled out. We agree with Salazar et al. that a caesarean section is indicated if la- 
bour starts while the mother is still on oral anticoagulants, as reversal of the mother's anticoagulation can be quickly achieved [16].

Although our series is one of the largest in the literature, the numbers are too small to come up with recommendations regarding the superiority of one anticoagulation regimen over the other in pregnant women with mechanical heart valves. It is apparent that no single anticoagulant regimen confers complete protection from thromboembolic phenomena in pregnancy.

\section{Conclusion}

In conclusion, there is to date no anticoagulant regimen that can be said to be entirely safe for use during pregnancy as there is a degree of risk with each regimen. In countries with poor socioeconomic background as in Tunisia, the patient's compliance is an important determining factor that needs to be considered before selection of a specific regimen. Use of oral anticoagulants may help to achieve better patient compliance. Our series, contrary to the earlier belief, had higher thrombotic complications leading to valve thrombosis while on acenocoumarol, but the numbers are too small to claim the superiority of the safety of one regime over the other. Further larger studies are needed to come up with evidence-based recommendations for the optimal management of such patients to reduce the maternal risks after mechanical heart valve replacement without compromising fetal outcome.

\section{References}

[1] Schafer, A.I. (1985) The Hypercoagulable States. Annals of Internal Medicine, 102, 814-828. http://dx.doi.org/10.7326/0003-4819-102-6-814

[2] Vitale, N., De Feo, M., De Santo, L.S., Pollice, A., Tedesco, N. and Cotrufo, M. (1999) Dose-Dependent Fetal Complications of Warfarin in Pregnant Women with Mechanical Heart Valves. Journal of the American College of Cardiology, 33, 1637-1641. http://dx.doi.org/10.1016/S0735-1097(99)00044-3

[3] Chan, W.S., Anand, S. and Ginsberg, J.S. (2000) Anticoagulation of Pregnant Women with Mechanical Heart Valves: A Systematic Review of the Literature. Archives of Internal Medicine, 160, 191-196. http://dx.doi.org/10.1001/archinte.160.2.191

[4] McLintock, C. (2011) Anticoagulant Therapy in Pregnant Women with Mechanical Prosthetic Heart Valves: No Easy Option. Thrombosis Research, 127, S56-S60. http://dx.doi.org/10.1016/S0049-3848(11)70016-0

[5] Sadler, L., McCowan, L., White, H., Stewart, L., Bracken, M. and North, R. (2000) Pregnancy Outcomes and Cardiac Complications in Women with Mechanical, Bioprosthetic and Homograft Valves. BJOG: An International Journal of Obstetrics \& Gynaecology, 107, 245-253. http://dx.doi.org/10.1111/j.1471-0528.2000.tb11696.x

[6] Hanania, G. (2001) Management of Anticoagulants during Pregnancy. Heart, 86, 125-126. http://dx.doi.org/10.1136/heart.86.2.125

[7] Vongpatanasin, W., Hullis, D.L. and Lange, R.A. (1996) Prosthetic Heart Valves. The New England Journal of Medicine, 335, 407-416. http://dx.doi.org/10.1056/NEJM199608083350607

[8] Hirsh, J., Fuster, V., Ansell, J. and Halperin, J.L. (2003) American Heart Association/American College of Cardiology Foundation Guide to Warfarin Therapy. Journal of the American College of Cardiology, 41, 1633-1652. http://dx.doi.org/10.1016/S0735-1097(03)00416-9

[9] Baroletti, S.A. and Goldhaber, S.Z. (2006) Heparin-Induced Thrombocytopenia. Circulation, 22, e355-e356. http://dx.doi.org/10.1161/CIRCULATIONAHA.106.632653

[10] Sbarouni, E. and Oakley, C.M. (1994) Outcome of Pregnancy in Women with Valve Prostheses. British Heart Journal, 71, 196-201. http://dx.doi.org/10.1136/hrt.71.2.196

[11] Hall, J.G., Pauli, R.M. and Wilson, K.M. (1980) Maternal and fetal Sequelae and Anticoagulation during Pregnancy. The American Journal of Medicine, 68, 122-140. http://dx.doi.org/10.1016/0002-9343(80)90181-3

[12] Ginsberg, J.S. and Hirsh, J. (1998) Use of Antithrombotic Agents during Pregnancy. Chest Journal, 114, 524S-530S. http://dx.doi.org/10.1378/chest.114.5_Supplement.524S

[13] Nassar, A.H., Hobeika, E.M., Essamad, A.H.M., Taher, A., Khalil, A.M. and Usta, I.M. (2004) Pregnancy Outcome in Women with Prosthetic Heart Valves. American Journal of Obstetrics \& Gynecology, 191, 1009-1013. http://dx.doi.org/10.1016/j.ajog.2004.05.064

[14] Khamooshi, A.J., Kashfi, F., Hoseini, S., Tabatabaei, M.B., Javadpour, H. and Noohi, F. (2007) Anticoagulation for Prosthetic Heart Valves in Pregnancy. Is There an Answer? Asian Cardiovascular and Thoracic Annals, 15, 493-496. http://dx.doi.org/10.1177/021849230701500609 
[15] van Leeuwen, Y., Rosendaal, F.R. and van der Meer, F.J.M. (2008) The Relationship between Maintenance Dosages of Three Vitamin K Antagonists: Acenocoumarol, Warfarin and Phenprocoumon. Thrombosis Research, 123, 225-230. http://dx.doi.org/10.1016/j.thromres.2008.01.020

[16] Salazar, E., Izaguirre, R., Verdejo, J.G., et al. (1996) Failure of Adjusted Doses of Subcutaneous Heparin to Prevent Thromboembolic Phenomena in Pregnant Patients with Mechanical Cardiac Valve Prostheses. Journal of the American College of Cardiology, 27, 1698-1703. http://dx.doi.org/10.1016/0735-1097(96)00072-1 